\title{
Unexpected decline in survival from amyotrophic lateral sclerosis/motor neurone disease
}

\author{
R B Forbes, S Colville, G W Cran, R J Swingler
}

J Neurol Neurosurg Psychiatry 2004;75:1753-1755. doi: 10.1136/jnnp.2003.024364

\begin{abstract}
Objectives: To describe survival of 1226 Scottish adults with amyotrophic lateral sclerosis/motor neurone disease (ALS) MND).

Methods: Ten year, prospective, population based disease register. Cox time dependent proportional hazards modelling for multivariate survival analyses.

Results: Median survival from onset was 25 months (interquartile range 16-34 months). In multivariate models we found an increased hazard with more recently diagnosed cases - that is, there was an unexpected decline in survival over the 10 year period (hazard ratio (HR) $1.06(95 \% \mathrm{Cl}$ 1.04 to 1.09). Positive effects on survival were demonstrated for longer time from onset to diagnosis (HR $0.38195 \% \mathrm{Cl}$ 0.33 to 0.42 ), assessment by a neurological specialist (HR 0.56 (95\% $\mathrm{Cl} 0.40$ to 0.77 ), and treatment with riluzole (HR 0.24 (95\% Cl 0.14 to 0.42 ). Poor prognosis was associated with bulbar onset (HR $1.25(95 \% \mathrm{Cl} 1.09$ to 1.46$)$ and a mixed lower and upper motor neurone syndrome (HR 1.23 (95\% Cl 1.01-1.49) and increasing age.

Conclusions: We found an unexpected decline in survival over the 10 year period, despite controlling for potential confounding variables. We would be cautious about overinterpreting these observations and suggest that further research is required to confirm or refute these findings.
\end{abstract}

$\mathrm{T}$ raditionally, average survival of patients with amyotrophic lateral sclerosis/motor neurone disease (ALS/ MND) is estimated to be two to three years from onset. In recent years the management of ALS/MND has changed, primarily through the use of riluzole-a glutamate antagonist shown to increase survival in randomised controlled trials $^{12}$ - and the introduction of nutritional support methods such as percutaneous gastrostomy (PEG) feeding tubes. ${ }^{3}$

Observational data have demonstrated improved survival in recently treated cases, particularly when treated with riluzole. ${ }^{45}$ However, a recent population based study from Rochester showed a non-significant trend towards reduced survival in people diagnosed since 1990 (compared with diagnoses made pre-1990). ${ }^{6}$ A population based survey in Ireland did not find evidence of increased survival over time, but that improved survival was associated with attendance at a multidisciplinary ALS clinic. ${ }^{7}$ To explore temporal trends in survival we studied the prognosis of 1226 Scottish adults diagnosed between 1989 and 1998.

\section{METHODS}

The Scottish Motor Amyotrophic Lateral Sclerosis/Motor Neurone Disease Register was a prospective study with multicentre research ethics approval. ${ }^{8}$ Potential cases of ALS/MND were identified from multiple sources. After notification, neurologists with experience of epidemiologic research scrutinised the medical records of each person for evidence consistent with a diagnosis of ALS. If a patient was felt to have a clinical diagnosis of ALS, they were eligible for inclusion in the register and were classified according to the modified World Federation criteria or the El Escorial criteria. For the first 614 patients (diagnosis made in 1989-93) we used modified World Federation of Neurology diagnostic criteria. ${ }^{8}$ For the following 612 patients (diagnosis made in 1994-98) the El Escorial criteria9 were used. Suspected ALS was included in the El Escorial group, so that those presenting with lower motor neurone syndromes (and no evidence at registration of upper neurone involvement) were included throughout the course of the ten year study. Autopsies, obtained in the course of usual clinical practice, provided neuropathological confirmation of the clinical diagnosis in 69 people. One other case had a clinical diagnosis of ALS but pathological examination demonstrated Alzheimer's pathology.

We recorded whether a patient attended a neurologist or non-neurologist, and if they had received riluzole by the time of registration. From case records, we obtained the time from onset to diagnosis, location of symptom onset (bulbar or spinal), and presence of isolated lower motor neurone syndrome or mixed upper and lower motor neurone syndrome. We received the dates of deaths from the Office of the Registrar General for Scotland on an annual basis. The last full year for which we have complete death notification is 1999.

Of the 1226 people with ALS, 130 had a hospital discharge coded for gastrostomy or endoscopic gastrostomy during the study period. We obtained these hospital discharge codes from a record linkage study, approved by the Scottish Office, with the Scottish Morbidity Records dataset.

\section{Survival analysis}

We investigated survival using Cox time dependent regression modelling. The following variables were considered: year of diagnosis, age at onset, bulbar onset (compared with spinal onset), lower motor neurone syndrome versus mixed upper and lower syndrome, assessment by a neurologist, usage of riluzole, time from onset to diagnosis, sex, family history of ALS/MND, PEG tube procedure, and diagnostic criteria used. Hazard ratios were calculated for each variable, and were significant at the 0.05 level if $95 \%$ confidence intervals did not include 1 . We derived the final model using a stepwise method. Factors which did not contribute to survival (at a significance level of $\mathrm{p}<0.05$ ) were omitted from the final model. In particular, a variable to discriminate people whose disease was diagnosed with the El Escorial criteria from those whose diagnosis was made with the modified World Federation criteria was removed from the final model as it did not contribute to survival. For the time

Abbreviations: ALS, amyotrophic lateral sclerosis; MND, motor neurone disease; $P E G$, percutaneous gastrostomy 
dependent covariates we estimated hazard ratios at 0,1 , and 2 years.

\section{RESULTS}

We registered 1226 people with ALS, mean age at onset 65.2 (SD 11.9) years for men and 67.7 (11.0) years for women. The overall median survival from onset of symptoms was 25 months (interquartile range 16-34 months). The mean time from onset to diagnosis was 15.1 months (95\% CI 14.0 to 16.2) with a median of 10 months (interquartile range 6.0$17.0)$. Five year survival was $11 \%$. A total of 58 people $(4.4 \%)$ survived more than eight years from onset of symptoms.

In the multivariate model (table 1), independent adverse effects on survival remained for increasing date of diagnosis (from 1989 to 1998) and increasing age at onset. Even if cases with pure lower motor neurone syndromes were excluded, year of diagnosis remained an independent predictor of adverse survival (table 1). Those with bulbar onset disease (median survival 20.4 months, interquartile range 14.2-30.4) had a worse survival compared with spinal onset disease (median survival 28.4 months, interquartile range 17.450.0). The presence of both upper and lower motor neurone signs (median survival 24.2 months, interquartile range 15.5-38.9) had a worse prognosis than a pure lower motor neurone syndrome (median survival 28.9 months, interquartile range 17.6-53.3). Sex or the presence of a family history of ALS/MND did not influence survival (table 1).

Improved survival was associated with a longer time from onset to diagnosis, assessment by a neurologist, and treatment with riluzole (table 1). Our final regression model was based upon 1048 patients (948 deaths, 92 censored) as the model excluded any case with one or more missing observations.

\section{DISCUSSION}

We identified an unexpected decline in survival during the ten year period 1989-98. The difference in median survival between those diagnosed in 1989-93 and those diagnosed in 1994-98 was 2.4 months (fig 1). Even when controlling for confounding variables, including a variable to take account of the different diagnostic criteria (data not shown), or eliminating lower motor neurone syndromes (table 1), we were unable to eliminate this difference in a multivariate regression model. Before drawing further conclusions it is important to re-examine our data and make comparisons with other studies of survival of patients with ALS/MND.

In agreement with previous studies we found that increasing age at onset ${ }^{10}$ and bulbar onset disease ${ }^{11}$ predicted adverse survival, while the absence of upper motor neurone
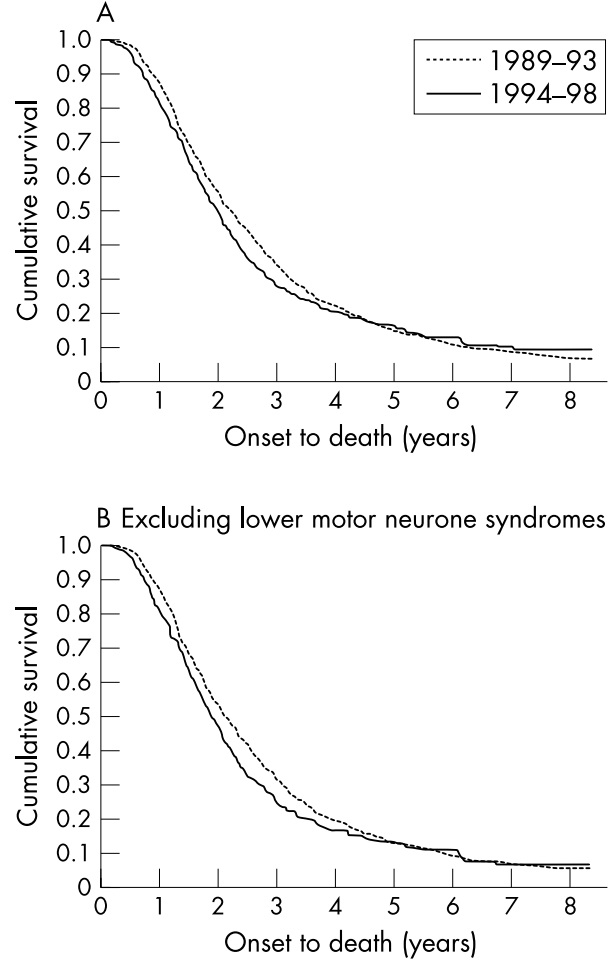

Figure 1 The Scottish Motor Amyotrophic Lateral Sclerosis/Motor Neurone Disease Register (ALS/MND) 1989-98. (A) Survival curves of patients diagnosed as having ALS in 1989-93 compared with those diagnosed in 1994-98, all cases. (B) Survival curves again comparing 1989-93 with 1994-98 but excluding lower motor neurone syndromes.

signs $^{12}$ at registration and a longer time from onset to diagnosis was associated with better outcome. As predicted from clinical trials, better prognosis was associated with use of riluzole. ${ }^{12}$

Some authors have reported better survival in women, ${ }^{13}$ while others have found better survival in men. ${ }^{12}$ In this study, the largest population based survival analysis to date, sex was not a significant predictor of survival (table 1).

In Scotland, over $80 \%$ of patients with ALS were managed by neurologists, and it appeared as if patients under their care had a better survival even when controlling for sex, bulbar onset, time from onset to diagnosis, and the use of riluzole. While this association may be spurious it does raise the possibility that the process of care for people with ALS is an important contributor to their survival.

Table 1 Multivariate Cox regression model of survival in amyotrophic lateral sclerosis: Scottish Motor Neurone Disease Register 1989-98

\begin{tabular}{|c|c|c|c|c|c|c|}
\hline & \multirow[b]{2}{*}{ B } & \multirow[b]{2}{*}{ SE } & \multirow[b]{2}{*}{$\mathbf{p}$} & \multicolumn{3}{|l|}{ Hazard ratio $(95 \% \mathrm{Cl})$} \\
\hline & & & & Time $=0$ years & Time $=1$ year & Time $=2$ years \\
\hline \multicolumn{7}{|l|}{ All cases } \\
\hline Year of diagnosis & 0.075 & 0.012 & 0.000 & 1.08 (1.05 to 1.10$)$ & & \\
\hline Increasing age at onset & 0.022 & 0.003 & 0.000 & 1.02 (1.01 to 1.03$)$ & & \\
\hline Bulbar onset & 0.232 & 0.074 & 0.002 & 1.25 (1.09 to 1.46$)$ & & \\
\hline Mixed upper and lower syndrome & 0.202 & 0.097 & 0.036 & 1.23 (1.01 to 1.49$)$ & & \\
\hline Specialist neurology assessment ${ }^{*}$ & -0.581 & 0.167 & 0.001 & $0.56(0.40$ to 0.77$)$ & $0.66(0.40$ to 0.78$)$ & 0.78 (0.66 to 0.92$)$ \\
\hline Use of riluzole* & -1.452 & 0.264 & 0.000 & 0.24 (0.14 to 0.42$)$ & $0.31(0.14$ to 0.39$)$ & $0.41(0.29$ to 0.58$)$ \\
\hline Time from onset to diagnosis* & -0.976 & 0.063 & 0.000 & 0.38 (0.33 to 0.42$)$ & $0.40(0.36$ to 0.45$)$ & $0.43(0.39$ to 0.48$)$ \\
\hline Sex ${ }^{*}$ & 0.189 & 0.110 & 0.085 & $1.21(0.97$ to 1.50$)$ & $1.11(0.97$ to 1.50$)$ & 1.02 (0.89 to 1.16$)$ \\
\hline \multicolumn{7}{|l|}{ Lower motor neurone syndromes excluded } \\
\hline Year of diagnosis & 0.047 & 0.013 & 0.000 & 1.05 (1.02 to 1.08$)$ & & \\
\hline
\end{tabular}

*These covariates have a time dependent component.

$\mathrm{SE}$, standard error. 
It is interesting that despite the introduction of riluzole and PEG we found a decline in average survival between 1989 and 1998. Clearly those who received riluzole had a survival advantage, but this effect was subsumed by a larger negative effect associated with increasing year of diagnosis. Since our register's inception, we are confident that ascertainment in our population has been near complete, and there is no evidence that we are identifying an increasing number of previously undetected patients with severe illness. Our method of ascertainment did not change in the ten year study, with patients accepted on the basis of a clinical diagnosis of ALS/MND. We emphasise that the diagnostic criteria have been applied following clinical diagnosis, and even when taking into account the two sets of criteria, or eliminating contentious lower motor syndromes a decline in survival appears to have been observed.

The recently reported data from Rochester suggested a trend towards poorer survival in recently diagnosed cases (pre-1990 v post-1990). ${ }^{6}$ Owing to their small sample size no conclusions could be drawn, but our observations add strength to the hypothesis that despite attempted therapeutic interventions there is an overall decline in expected survival. A recent population based survey from Ireland reported increased survival (for those with bulbar onset disease) attending a specialist ALS clinic. Using Kaplan-Meier methods they compared 1996-1998 against 1999-2000 and found no change in length of survival over time. ${ }^{7}$

It is still unclear whether our observed reduction in survival over time is real. It is possible that our apparent declining survival is a statistical artefact due to confounding variables. However, if real, it is difficult to explain the counterintuitive finding of declining survival. Although "right to die" court cases have been highlighted in the media in the UK, we have no evidence from Scotland that increasing numbers of people with ALS are executing advanced directives or that euthanasia is being practised outwith present laws. While one may speculate that therapeutic nihilism could lead neurologists or other medical practitioners to withdraw treatment or nutritional support to hasten death, we are observing increasing use of riluzole and PEG tubes in our population indicating that ALS is being seen as a potentially treatable disorder. The apparent decline in survival could reflect increased patient autonomy, as patients, when given the choice, may opt for less aggressive therapy in the latter stages of their disease. A more palliative approach may reduce the duration of their survival, but this situation would be tolerable if the quality of the shorter life span was increased. However, until our observations are reproduced we would be cautious about making any further inferences about the care of people with ALS.

\section{ACKNOWLEDGEMENTS}

We wish to thank the Scottish MND Register Research Group Collaborators and the Family Care Officers/Nurse Specialists of the Scottish Motor Neurone Disease Association, Mr S Ogston and Dr
C Lien, Ninewells Hospital and Medical School, Dundee, and Drs Andrew Chancellor and R Davenport for initial work establishing the Scottish Motor Neurone Disease Register.

\section{Authors' affiliations}

R B Forbes, S Colville, R J Swingler, For The Scottish Motor Neurone Disease Register, Department of Neurology, Ninewells Hospital and Medical School, Dundee, Scotland, UK

G W Cran, Department of Epidemiology and Public Health, Queens University, Belfast, Northern Ireland, UK

This work was funded by the Scottish Motor Neurone Disease Association

Competing interests: Aventis plc (manufacturer of riluzole) has sponsored RBF, SC and RJS for attending scientific conferences. GC has no competing interests.

Correspondence to: Dr R B Forbes, Department of Neurology, Royal Victoria Hospital, Belfast BT12 6BA, Northern Ireland, UK; Raeburnforbes@aol.com

Received 18 August 2003

In revised form 24 March 2004

Accepted 24 March 2004

\section{REFERENCES}

1 Bensimon G, Lacomblez L, Meininger V. A controlled trial of riluzole in amyotrophic lateral sclerosis. ALS/Riluzole Study Group. N Engl J Med 1994;330:585-91.

2 Lacomblez L, Bensimon G, Leigh PN, et al. Dose-ranging study of riluzole in amyotrophic lateral sclerosis. Amyotrophic Lateral Sclerosis/Riluzole Study Group II. Lancet 1996;347:1425-31

3 Mazzini L, Corra T, Zaccala M, et al. Percutaneous endoscopic gastrostomy and enteral nutrition in amyotrophic lateral sclerosis. J Neurol 1995;242:695-8.

4 Turner M, Bakker M, Sham P, et al. The King's Database 1990-2000: an analysis of the effect on survival of interventions in ALS. Amyotroph Lateral Scler Other Motor Neuron Disord 2001;1(suppl 2):43.

5 Traynor BJ, Alexander M, Corr B, et al. An outcome study of riluzole in amyotrophic lateral sclerosis - a population-based study in Ireland, 19962000. J Neurol 2003;250:473-9.

6 Sorenson E, Stalker A, Kurland L, et al. Amyotrophic lateral sclerosis in Olmstead County, Minnesota, 1925-1998. Neurology 2002:59:280-2.

7 Traynor BJ, Alexander M, Corr B, et al. Effect of a multidisciplinary amyotrophic lateral sclerosis (ALS) clinic on ALS survival: a population based study 1996-2000. J Neurol Neurosurg Psychiatry 2003;74:1258-61.

8 Anonymous. The Scottish Motor Neurone Disease Register: a prospective study of adult onset motor neurone disease in Scotland. Methodology, demography, and clinical features of incident cases in 1989. J Neurol Neurosurg Psychiatry 1992;55:536-41.

9 Brooks BR. El Escorial World Federation of Neurology criteria for the diagnosis of amyotrophic lateral sclerosis. Subcommittee on Motor Neuron Diseases/Amyotrophic Lateral Sclerosis of the World Federation of Neurology Research Group on Neuromuscular Diseases and the El Escorial "Clinical limits of amyotrophic lateral sclerosis" workshop contributors. J Neurol Sci 1994; 124(suppl):96-107.

10 Eisen A, Schulzer M, MacNeil M, et al. Duration of amyotrophic lateral sclerosis is age dependent. Muscle Nerve 1993;16:27-32.

11 Rosen AD. Amyotrophic lateral sclerosis. Clinical features and prognosis. Arch Neurol 1978;35:638-42.

12 Norris F, Shepherd R, Denys E, et al. Onset, natural history and outcome in idiopathic adult motor neuron disease. J Neurol Sci 1993; 1 18:48-55.

13 Caroscio JT, Mulvihill MN, Sterling R, et al. Amyotrophic lateral sclerosis. Its natural history. Neurol Clin 1987;5:1-8. 